\title{
Oxidative Stress Alterations in the Bladder of a Short-period Type 2 Diabetes Rat Model: Antioxidant Treatment Can Be Beneficial for the Bladder
}

\author{
PANAGIOTA TSOUNAPI ${ }^{1}$, MASASHI HONDA ${ }^{1}$, KATSUYA HIKITA ${ }^{1}$, \\ NIKOLAOS SOFIKITIS ${ }^{2}$ and ATSUSHI TAKENAKA ${ }^{1}$ \\ ${ }^{1}$ Division of Urology, Department of Surgery, Tottori University Faculty of Medicine, Yonago, Japan; \\ ${ }^{2}$ Department of Urology, University of Ioannina School of Medicine, Ioannina, Greece
}

\begin{abstract}
Background/Aim: A short-period type 2 diabetes model was established in order to identify changes in oxidative stress in the bladder at the initiation of the disease. The effect of antioxidant treatment was examined. Materials and Methods: Diabetes was induced in adult male Wistar rats with a single dose of streptozotocin $(40 \mathrm{mg} / \mathrm{kg} ;$ i.p.). Diabetic animals were then randomly separated into three groups: No treatment (DM), resveratrol treatment, and taurine treatment, and fed with a high-fat diet. Age-matched non-diabetic animals were used and fed with normal diet (control). Two weeks later, animals were sacrificed and bladders were processed for histological evaluation, and further analysis for oxidative stress markers. Results: The body weight of all diabetic animals was significantly lower compared to the controls. The DM group demonstrated a significantly higher bladder weight to body weight ratio compared to the control. The bladder in the DM group demonstrated abruption of the mucosa from the muscularis and edema in the transitional epithelium. Bladders from the resveratrol-, and taurinetreated groups did not demonstrate these histological alterations. The level of malondialdehyde (MDA) in the bladder was significantly higher in the DM group compared to all other groups. Immunohistochemistry showed that diabetes induced a moderate to strong expression of oxidative stress markers MDA and 4-hydroxynonenal, and DNA oxidative stress marker 8-deoxyguanosine in the DM group
\end{abstract}

This article is freely accessible online.

Correspondence to: Masashi Honda, MD, Ph.D., Division of Urology, Tottori University, Faculty of Medicine, 36-1 Nishimachi, Yonago, 683-8503, Japan. Tel: +81 859386607, Fax: +81 859386609, e-mail: honda@med.tottori-u.ac.jp

Key Words: Antioxidants, bladder, diabetes, oxidative stress, immunohistochemistry. compared to the other groups. Conclusion: Prompt diagnosis and treatment of diabetes is crucial in regard to disease progression. Specifically, in the bladder it appears that both mild damage at the structural level, as well as oxidative damage at the molecular level may be prevented by antioxidant treatment.

According to the International Diabetes Federation Guideline Development Group (2014), in 2011, there were 336 million diabetic patients around the world (1). Additionally, the same report refers that this number is expected to reach 552 million by 2030 . It is not so difficult to imagine this rise of diabetes incidence, considering that the WHO global report on diabetes published in 2016 outlined that 422 million adults were living with diabetes in 2014 globally, compared to 108 million in 1980 (2). The age-standardized prevalence of diabetes around the world has, in fact, almost doubled from $4.7 \%$ to $8.5 \%$ since 1980 among the adult population. Type 2 diabetes accounts for up to $95 \%$ of all cases of diabetic patients (3). Type 2 diabetes has several causes: genetics and lifestyle are the most important ones. A combination of these factors can cause insulin resistance, when the body does not use insulin as well as it should. In general, as countries become richer, people eat a more sugarand fat-rich diet and are less physically active; as a result, the incidence of diabetes rises. On average, nearly $8 \%$ of adults living in high-income countries have diabetes. It is, however, upper-middle and middle-income countries that have the highest prevalence of diabetes; over $10 \%$ of adults in these countries have this condition (2).

The connection between diabetes and oxidative stress has been reported. The hypothesis is that oxidative stress, through the production of superoxide, is the common pathogenic factor resulting in insulin resistance, B-cell dysfunction, impaired glucose tolerance and ultimately type 2 diabetes mellitus (4). Furthermore, this mechanism has been implicated as the underlying cause of both the macrovascular 
and microvascular complications associated with type 2 diabetes mellitus (5). Therefore, it would be reasonable to consider that the reduction of oxidative stress might be beneficial for patients with type 2 diabetes mellitus and those who are at risk of developing diabetes.

In the present study, we aimed to investigate the diabetesinduced alterations that occur in the bladder during the initial stages of the disease. Additionally, we treated animals with two different antioxidants to study if diabetes-induced damage in the bladder can be reversed.

The two antioxidants that we employed for our study were resveratrol and taurine. Taurine is an amino sulfonic acid which is essential for cardiovascular function, and development and function of skeletal muscle, the retina, and the central nervous system. Taurine plays an important role in several essential biological processes such as membrane stabilization and immunity; it has anti-oxidant and antiinflammatory effects (6).

Resveratrol is a non-flavonoid polyphenol compound which can be found in abundance in fruits such as berries, red grapes and in peanuts (7). Additionally, resveratrol protects against a wide range of aging-related diseases, such as type 2 diabetes, cardiovascular diseases, and neurodegenerative diseases including Alzheimer's disease (8). Moreover, the antioxidant properties of resveratrol have been demonstrated (9-11).

\section{Materials and Methods}

Ethics statement. The animal experiments were approved by the Tottori University Committee for Animal Experimentation (protocol approval number: 16-Y-23) and performed according to the Committee's guidelines for the care and handling of laboratory animals, which comply with the "Guidelines for Proper Conduct of Animal Experiments" developed by the Science Council of Japan.

Chemicals. Resveratrol, streptozotocin and taurine were purchased from Sigma-Aldrich (St. Louis, MO, USA). All chemicals not otherwise mentioned were of reagent grade and commercially available.

Animal model for type 2 diabetes. Forty, six-week-old male Wistar rats (180-210 g) (Japan SLC, Inc., Shizuoka, Japan) were used for this study. The animals were placed in polycarbonate cages in a room with a 12-h day-night cycle, constant room temperature of $22 \pm 2^{\circ} \mathrm{C}$ and humidity of $50-65 \%$. The animals were allowed to adjust for 2 weeks. Upon reaching 8 weeks of age, the animals were randomly separated into two groups. One group was the control and consisted of 10 animals. The control animals received a single

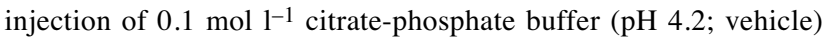
intraperitoneally (i.p.). The remaining 30 animals were included in the larger group of diabetes.

The protocol of the induction of diabetes type 2 was performed according to the method described by Dong et al. (12) with minor modifications. In brief, the 30 animals of the diabetes group followed a 12-h fasting period, and subsequently a combinational procedure which included a single injection with streptozotocin (40 $\left.\mathrm{mg} \mathrm{kg}^{-1}\right)$ i.p. dissolved in $0.1 \mathrm{~mol} \mathrm{l}^{-1}$ citrate phosphate. The diabetic animals followed a high-fat diet (HFD; EPS Research Diet, Japan, Product No: D12492) with $60 \%$ of calories derived from fat) for 14 days, while the animals in the control group were fed normal rodent chow during the same period. The induction of diabetes was confirmed by measuring the glucose in the urine with Pretest 3all (Wako Pure Chemical, Osaka, Japan) 2 days after the streptozotocin injection. The animals that demonstrated a test result of more than +++ , equivalent to $\geq 500 \mathrm{mg} \mathrm{dl}^{-1}$ urinary glucose, were included in the study. The induction of diabetes was successful in all animals. These 30 diabetic animals were divided randomly into three groups: One diabetic group (DM group, $\mathrm{n}=10$ ) was treated daily with $0.5 \mathrm{ml}$ saline i.p.; another group of diabetic animals received daily oral treatment with resveratrol at a dose of $10 \mathrm{mg} \mathrm{kg}^{-1}$ in aqueous suspension $(n=10)$; and the third diabetic group was daily administered $1 \mathrm{~g} \mathrm{~kg}^{-1}$ taurine diluted in saline, i.p. $(\mathrm{n}=10)$. Treatment with antioxidants or saline started 1 day after the confirmation of diabetes induction and was performed once every day for 14 days. The control group received $0.5 \mathrm{ml}$ of saline i.p. daily for the same period. During the experimental period, the animals were kept under identical conditions and had free access to food (normal diet or HFD) and fresh drinking water.

At the completion of the experimental period of 14 days, all animals were weighed and subsequently anaesthetized with pentobarbital $\left(50 \mathrm{mg} \mathrm{kg}^{-1}\right)$ i.p. Blood samples were aspirated from the inferior vena cava, serum was separated, frozen in liquid nitrogen and stored at $-80^{\circ} \mathrm{C}$. Additionally the bladders were collected. One part of the tissue was fixed in formalin (10\%) and the remaining tissue was frozen in liquid nitrogen and stored at $-80^{\circ} \mathrm{C}$ for further use. Afterwards, the animals were sacrificed with an overdose of pentobarbital (100 $\mathrm{mg} \mathrm{kg}^{-1}$, i.p.).

Serum glucose level in animals. The measurement of the glucose level in the serum was performed using an enzymatic method (mutarotase-glucose oxidase, Autokit Glucose; Wako Pure Chemical, Osaka, Japan). The method was carried out according to the manufacturer's instructions. The animals did not receive food $12 \mathrm{~h}$ before sacrifice.

Levels of oxidative stress markers in the bladder of the experimental animals. In order to determine the levels of diabetes-induced oxidative stress in the bladder tissue, the concentration of malondialdehyde (MDA) was measured as a representative and reliable marker for evaluation of lipid peroxidation (13). A commercially available kit was used (NWLSSTM Malondialdehyde Assay; Northwest Life Science Specialties, LLC, Vancouver, WA, USA), and the method was performed as previously described (14). The MDA concentrations were normalized by the protein content. Protein was determined using a commercial kit (Protein Assay Rapid Kit Wako, Wako Pure Chemical, Osaka, Japan).

Samples preparation for histopathological analysis. After fixation in $10 \%$ formalin, the bladder tissues were embedded in paraffin. Cross sections $(5 \mu \mathrm{m})$ were cut from the paraffin blocks. Sections were de-paraffinized, gradually hydrated, and examined by hematoxylin and eosin staining for changes or abnormalities in the histological structure of the bladder. Each section was viewed under light microscopy at $\times 400$ magnification. Histological examinations were performed by a pathologist blinded to the experiment who qualitatively evaluated bladder histology. The qualitative evaluation 
Table I. The general characteristics of the experimental animals, serum glucose level and malondialdehyde (MDA) level in the bladder of rats from the control group (fed normal chow and treated with saline daily for 14 days), DM group (diabetic rats fed high-fat diet, treated with saline daily for 14 days), resveratrol-treated group (diabetic rats fed high-fat diet, treated with $10 \mathrm{mg} \mathrm{kg}^{-1}$ resveratrol daily for 14 days), and taurine-treated group (diabetic rats fed high-fat diet, treated with $1 \mathrm{~g} \mathrm{~kg}^{-1}$ taurine daily for 14 days).

Animal group $(\mathrm{n}=10)$

\begin{tabular}{lrrrr} 
& \multicolumn{1}{c}{ Control } & DM & Resveratrol & Taurine \\
\cline { 2 - 5 } Parameter & $402.160 \pm 2.992$ & $309.209 \pm 6.371^{*}$ & $336.570 \pm 3.829^{* \#}$ & $343.165 \pm 2.459^{* \#}$ \\
BW $(\mathrm{g})$ & $0.180 \pm 0.008$ & $0.230 \pm 0.006^{*}$ & $0.196 \pm 0.009^{\#}$ & $0.180 \pm 0.009^{\#}$ \\
BLW $(\mathrm{g})$ & $4.466 \pm 0.165$ & $7.465 \pm 0.184^{*}$ & $5.865 \pm 0.236^{* \#}$ & $5.257 \pm 0.237^{* \#}$ \\
BLW/BW $\left(\times 10^{-4}\right)$ & $88.927 \pm 3.561$ & $414.958 \pm 13.999^{*}$ & $319.975 \pm 9.912^{* \#}$ & $304.447 \pm 13.328^{* \#}$ \\
Serum glucose $(\mathrm{mg} / \mathrm{dl})$ & $0.878 \pm 0.145$ & $2.701 \pm 0.327^{*}$ & $1.450 \pm 0.189^{* \#}$ & $0.978 \pm 0.163^{\#}$ \\
Bladder MDA $(\mathrm{mmol} / \mathrm{mg}$ protein) &
\end{tabular}

BW: Body weight; BLW: bladder weight. Significantly different at $p<0.05$ compared to: *control group; \#DM group.

included a scale of structural damage defined as: None, when no damage was observed; mild, when abruption of the lamina propria from the transitional epithelium and edema in the substantial epithelium were observed; and severe, when abruption of the lamina propria from the transitional epithelium, edema in the substantial epithelium, degeneration of the submucosa and lesions in muscular layer were observed. All samples were evaluated from each group.

Immunohistochemistry for lipid peroxidation markers 4-hydroxy-2nonenal (4-hNE), MDA and DNA oxidative stress marker 8-hydroxy2-deoxyguanosine $(8-\mathrm{OHdG})$. All the samples from each group were stained and evaluated. The samples were deparaffinized in xylene (three times $\times 3 \mathrm{~min}$ ), rehydrated in graded alcohols $(100 \%$, twice $\times 3 \mathrm{~min} ; 95 \%$, twice $\times 3 \mathrm{~min} ; 70 \%$, once $\times 3 \mathrm{~min}, 60 \%$ once $\times 3 \mathrm{~min}$ ) and distilled water for $5 \mathrm{~min}$ (once). Then the sections were subjected to antigen retrieval using a microwave in a $10 \mathrm{mM}$ citrate buffer (pH 6.0) for $10 \mathrm{~min}$ (this step was not performed for MDA detection) followed by returning to room temperature using running tap water. For the evaluation of $8-\mathrm{OHdG}$, the sections were incubated in Triton X-100 for $10 \mathrm{~min}$ at room temperature and afterwards the sections were washed three times with phosphatebuffered saline. Accordingly, the samples were incubated in $0.3 \%$ $\mathrm{H} 2 \mathrm{O} 2$ for $15 \mathrm{~min}$ at room temperature then washed with phosphatebuffered saline three times for $5 \mathrm{~min}$. For saturation of non-specific binding sites, blocking was performed using $1.5 \%$ normal horse serum (Peroxidase mouse IgG, PK-4002, Vectastain; Vector Laboratories, Burlingame, CA, USA) for $30 \mathrm{~min}$ in a humidified chamber at room temperature. Each primary antibody was diluted and applied and the samples were incubated overnight at $4^{\circ} \mathrm{C}$ in a humidified chamber. The primary antibodies used were: Mouse monoclonal antibody against 4-hNE (1:10; Japan Institute for the control of Aging, Shizuoka, Japan), mouse antibody against MDA (1:50; NOF Corporation, Tokyo, Japan), and mouse monoclonal antibody against $8-\mathrm{OHdG}$ (1:15; Japan Institute for the control of Aging). The next day, after washing three times with phosphatebuffered saline for $5 \mathrm{~min}$ each, biotinylated horse anti-mouse $\mathrm{IgG}$ (1:200) was applied to the tissue sections which were then incubated for $30 \mathrm{~min}$ in humidified chamber at room temperature. Immunoreaction was performed with an avidin-biotin complex alkaline phosphatase kit (Vectastain; Vector Laboratories). The sections were counterstained with hematoxylin. Negative control sections were processed in the absence of primary antibody. All samples were evaluated for specificity or background staining levels in comparison to the negative control sections.

Data analysis and statistical evaluation. Data are presented as means \pm S.E.M. of 10 separate determinations from each group. A statistical comparison of differences between groups was performed using analysis of variance and Fisher's multiple comparison tests. A $p$-value smaller than 0.05 was considered statistically significant.

\section{Results}

General features of the animals. All the animals survived until the completion of the study. Fourteen days of diabetes resulted in significantly lower body weight in these animals compared to the control animals (Table I). Treatment with taurine or resveratrol did not result in any significant change in the body weight of the treated diabetic animals compared to the DM group, but body weight was significantly lower compared to the control group. The bladder weight in the DM group was significantly increased compared to the control (Table I). In the treatment groups the bladder weights were significantly different compared to those of the DM group, while there was no statistically significant difference from those of the control group. Because the development of the animals during the 14 days of the experiment was different, we further evaluated the ratio of the bladder weight to the body weight of the animals. Our results indicated that the ratio in the DM group was significantly higher compared to the other three groups (Table I). In the resveratrol-, and taurine-treated groups, the ratio was significantly higher compared to the control group $(p=0.0001$ and $p=0.0135$, respectively).

The serum glucose level in experimental animals. The serum level of glucose in all diabetic animals was significantly increased compared to that of the control group. Treatment with resveratrol or taurine did not affect hyperglycemia but significantly reduced the serum glucose level compared to the respective values of the DM group (Table I). 

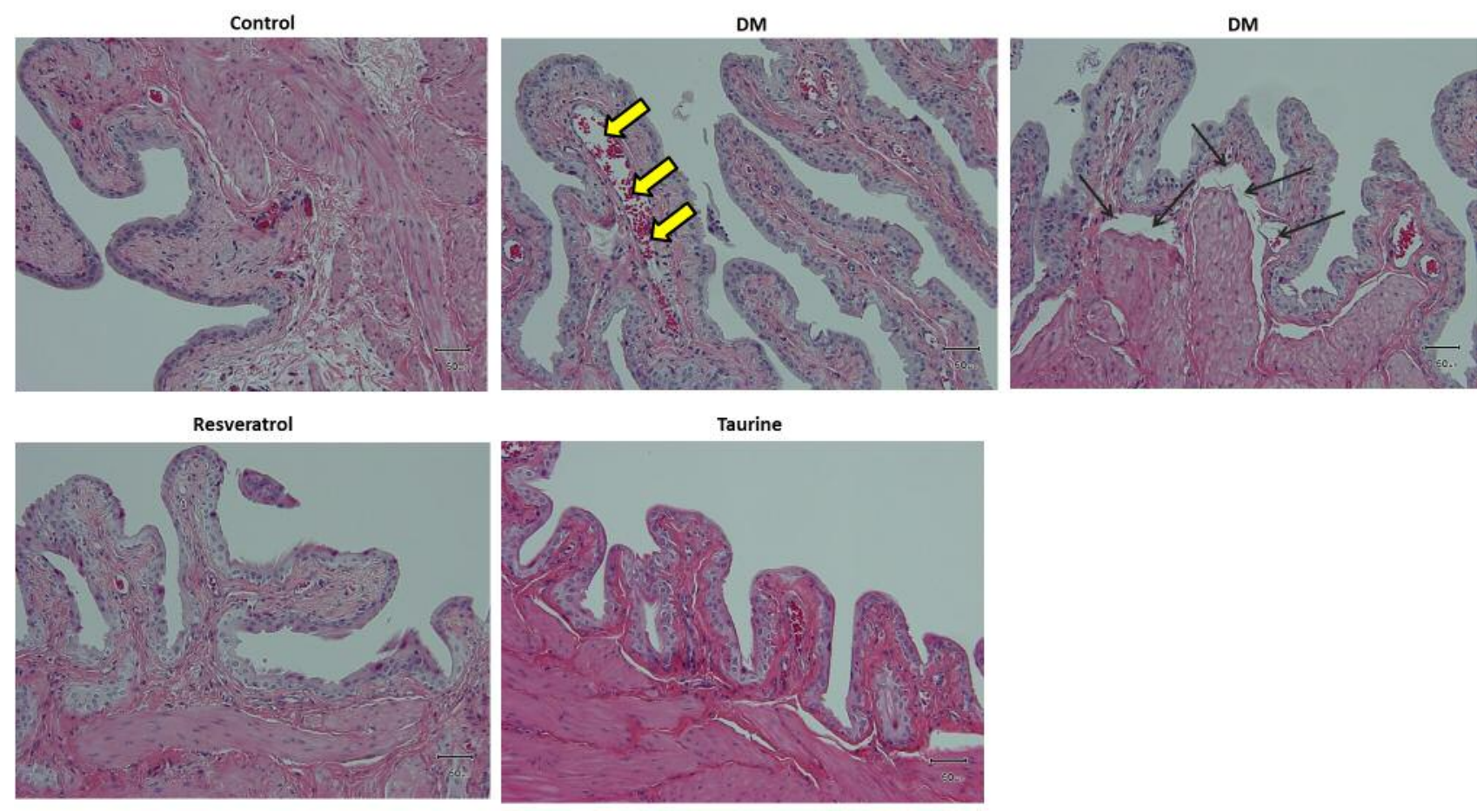

Figure 1. Representative hematoxylin-eosin-stained bladder tissue from all groups. Untreated diabetes induced mild damage to the bladder tissue. The yellow arrows indicate edema in the transitional epithelium and the black arrows indicate abruption of the mucosa from the muscularis. Control group: Fed normal chow and treated with saline daily for 14 days. DM group: Diabetic rats fed with high-fat diet and treated with saline daily for 14 days. Resveratrol group: Diabetic rats fed with high-fat diet and treated with $10 \mathrm{mg} \mathrm{kg-1}$ resveratrol daily for 14 days. Taurine: Diabetic rats fed with high-fat diet and treated with $1 \mathrm{~g} \mathrm{~kg}^{-1}$ taurine daily for 14 days. Original magnification: $\times 200$. Scale bar=50 $\mu \mathrm{m}$.

Levels of lipid peroxidation in the bladder of the experimental animals. Diabetes significantly increased the level of MDA in the bladder compared to the control: the MDA level in the DM group was three times higher compared to the control (Table I). Treatment with resveratrol or taurine significantly reduced the MDA level in the bladder compared to that of the DM group.

Histological alterations and oxidative stress markers in bladder tissue. Histological evaluation of the bladder demonstrated mild damage of the tissue in the DM group such as abruption of the mucosa from the muscularis as well as edema in the transitional epithelium. None of these alterations were observed in the treatment groups (Figure 1).

Fourteen days of diabetes without treatment in the DM group induced moderate to strong expression of oxidative stress markers 4-hNE (Figure 2), MDA (Figure 3), and DNA oxidative stress marker $8-\mathrm{OHdG}$ (Figure 4) compared with the other three groups. In the DM group, these oxidative stress markers were localized in the transitional epithelium and the mucosa, as well as the muscular area. Treatment with resveratrol or taurine inhibited the expression of these oxidative stress markers to a significant degree. In treatment groups, 4-hNE was present only in the transitional epithelium at low expression; MDA was not expressed in the tissue, and 8-OHdG was present in the transitional epithelium at low to moderate expression.

\section{Discussion}

Type 2 diabetes can affect many aspects of a patient's overall health, but if diagnosed and treated at an early stage, progression can be prevented and the condition can be reversible.

In the present study, we designed a short-period animal mode of type 2 diabetes in order to study oxidative stressrelated alterations in the bladder in the very early stages of the disease, and investigate to which extent antioxidant treatment might be beneficial in preventing damage of the tissue. Our results indicate that the histological changes that are induced by diabetes in the initiation of the disease appear to be mild. On the other hand, oxidative stress markers, even at this early stage, were highly expressed and present in the bladder. Both resveratrol and taurine had a significantly 


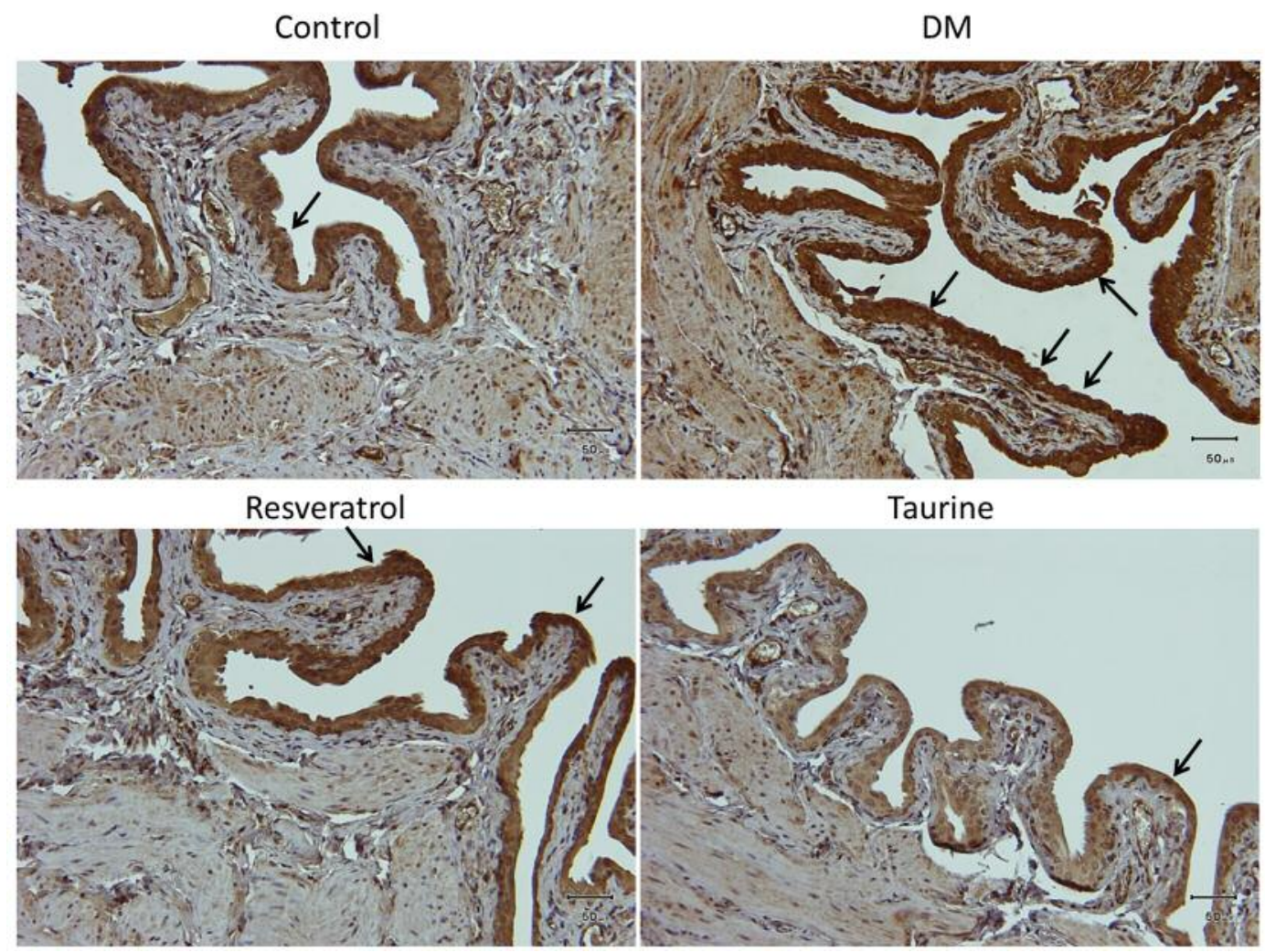

Figure 2. Lipid peroxidation marker 4-hydroxy-2-nonenal is strongly expressed in the urothelium in the bladder of DM animals, and appeared to be mildly expressed in the submucosa and the muscularis. Arrows indicate 4-hydroxy-2-nonenal -positive cells stained with brown color. Control group: Fed normal chow and treated with saline daily for 14 days. DM group: Diabetic rats fed with high-fat diet and treated with saline daily for 14 days. Resveratrol group: Diabetic rats fed with high-fat diet and treated with $10 \mathrm{mg} \mathrm{kg}^{-1}$ resveratrol daily for 14 days. Taurine: Diabetic rats fed with high-fat diet and treated with $1 \mathrm{~g} \mathrm{~kg}^{-1}$ taurine daily for 14 days. Original magnification: $\times 200$. Scale bar=50 $\mu \mathrm{m}$.

positive effect on the histological abnormalities induced by diabetes, presenting a histological profile of the bladder closer to that of the control group; therefore, antioxidant treatment may be of value. Additionally, lipid peroxidation levels as evaluated by the concentration of MDA in the bladder as well as the expression and localization of markers of oxidative stress and DNA oxidative damage in the bladder were significantly reduced by resveratrol, and by taurine treatment compared to the DM group. This gives further evidence that diabetes-induced oxidative stress in the bladder is related to histological damage in the bladder. Consequently, down-regulation of oxidative stress levels may result in the improvement of bladder histology.

Ito and co-workers in their review on the potential usefulness of taurine in diabetes discuss how taurine can provide cytoprotection through its ability to inhibit MDA and advanced glycation end products (15). Additionally, taurine regulates a variety of cellular functions, including antioxidant, modulation of ion movement or of neurotransmitters, and osmoregulation (15). We demonstrated the protective effects of taurine in endothelium dysfunction of the aorta in a rat model of type 1 diabetes (16), as well as in the male urogenital system (17-19). Taurine in the present model of type 2 diabetes may have acted by enhancing the antioxidant mechanisms of the bladder and by inhibiting lipid peroxidation, thereby preventing damage of the tissue. Resveratrol also acted in the same way, by reducing the MDA level in the bladder and further protecting the tissue. Resveratrol has been found to have a variety of beneficial effects, including: improvement of glucose homeostasis, reduction of insulin resistance, protection of pancreatic $\beta$-cell, advancement of insulin secretion and amelioration of metabolic disorders (20). These effects activated by resveratrol are correlated to the ability of this compound to increase expression/activity of $5^{\prime}$ adenosine monophosphate-activated protein kinase and sirtuin 1 in various tissues of diabetic individuals, as Szkudelski and Szkudelska describe in their review (20). Furthermore, resveratrol has anti-oxidant and anti-inflammatory properties that seem to play a role in its action in diabetic animal models (20). Although both antioxidants had a beneficial effect in 

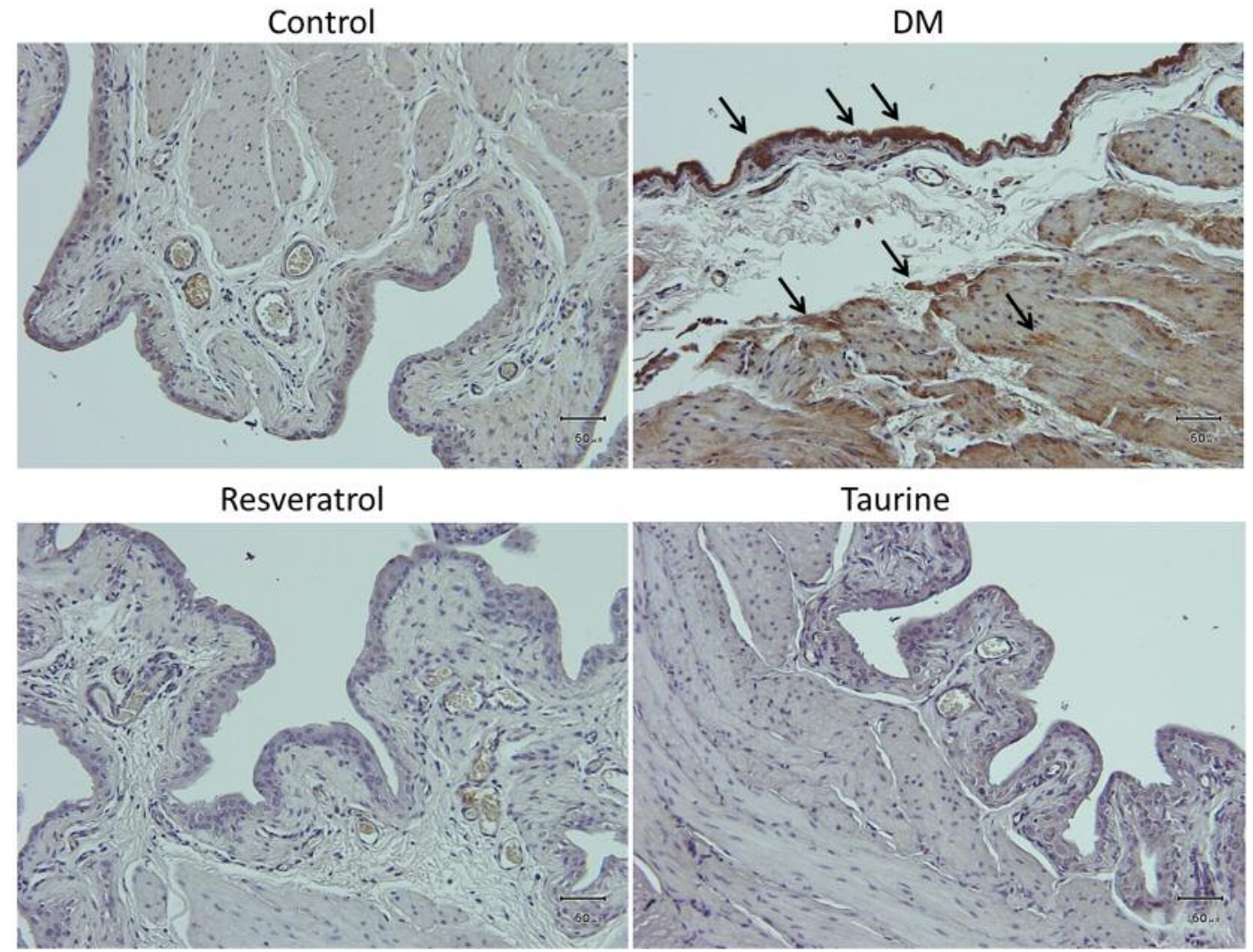

Figure 3. Malondialdehyde, a marker of lipid peroxidation, is mildly expressed in the urothelium and abrupt submucosa in the bladder of diabetic rats. Arrows indicate the malondialdehyde-positive cells stained with brown color. Control group: Fed normal chow and treated with saline daily for 14 days. DM group: Diabetic rats fed with high-fat diet and treated with saline daily for 14 days. Resveratrol group: Diabetic rats fed with high-fat diet and treated with $10 \mathrm{mg} \mathrm{kg}^{-1}$ resveratrol daily for 14 days. Taurine: Diabetic rats fed with high-fat diet and treated with $1 \mathrm{~g} \mathrm{~kg}^{-1}$ taurine daily for 14 days. Original magnification: $\times 200$. Scale bar $=50 \mu \mathrm{m}$.

lowering the glucose level compared to the DM group, they did not affect hyperglycemia, which can be further translated into meaning that antioxidants cannot replace anti-diabetic medication but may act as a supplementary therapy for diabetes-induced bladder dysfunction.

In previous studies by Ustuner and co-workers (21) and Aybek and co-workers (22), treatment with vitamin $\mathrm{E}$ in rats with type 2 diabetes had a beneficial effect on bladder histology $(21,22)$. In Ustuner et al.'s study, vitamin $\mathrm{E}$ at a dose of $40 \mathrm{mg} \mathrm{kg}^{-1}$ significantly reduced apoptosis, MDA and glutathione peroxidase, and on the other hand, increased the levels of antioxidant enzymes catalase and superoxide dismutase when compared to non-treated diabetic animals; all these alterations further protected the uroepithelial cells of the bladder in diabetes (21). In the study of Aybek et al., $50 \mathrm{IU} \mathrm{kg}{ }^{-1}$ of vitamin $\mathrm{E}$ was shown to significantly inhibit lipid peroxidation as demonstrated by reduction of levels of MDA and glutathione peroxidase compared to the non-treated diabetic animals (22). These changes further resulted in reduced collagen deposition in young and older diabetic animals treated with vitamin E (22). Our study, whereby, antioxidant supplementation reduced oxidative stress and resulted in a better histological pattern are consistent with their findings. Another study by $\mathrm{Ha}$ and co-workers demonstrated that cyanidin-3-O- $\beta$-D-glucopyranoside, a free-radical scavenger, by inhibiting apoptosis, reducing DNA oxidative damage and enhancing the antioxidant system of the bladder, significantly improved maximal detrusor pressure and restored the contractility of the tissue in a diabetic animal model (23). Jiang and co-workers also showed that alpha-lipoic acid, an essential component of mitochondria oxidative metabolism, significantly up-regulated expression of bladder nerve growth factor, reduced oxidative stress, and further normalized the bladder function in a diabetic model (24).

\section{Conclusion}

The prompt diagnosis of diabetes can be crucial for the progression of the disease. Specifically, in the bladder, it appears that both mild damage at the structural level, as well 


\section{Control}

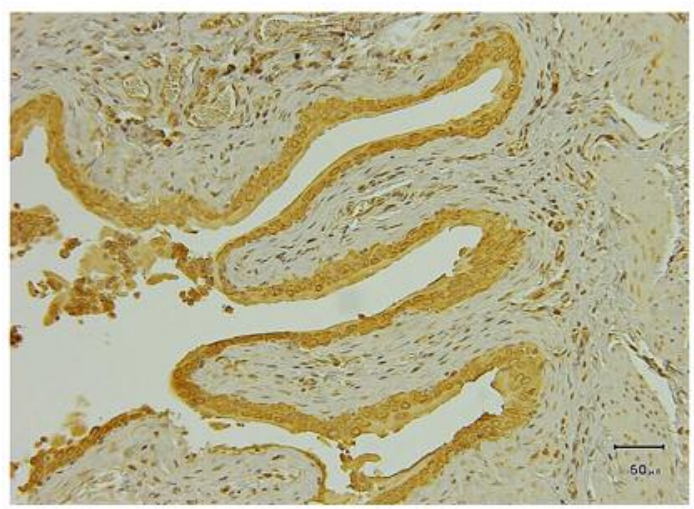

Resveratrol

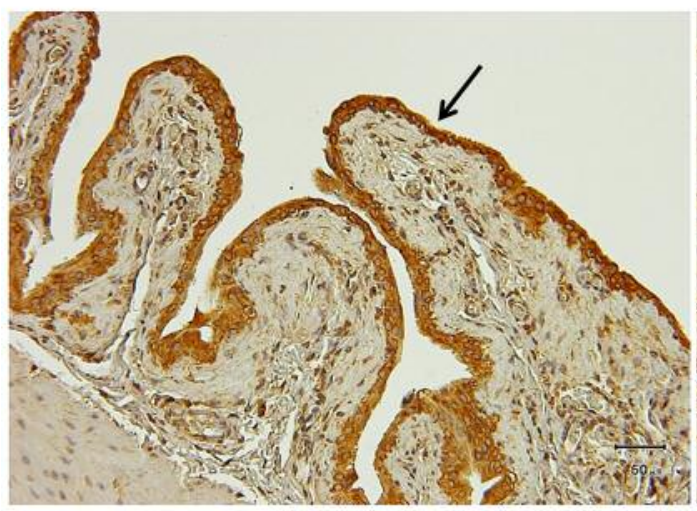

DM

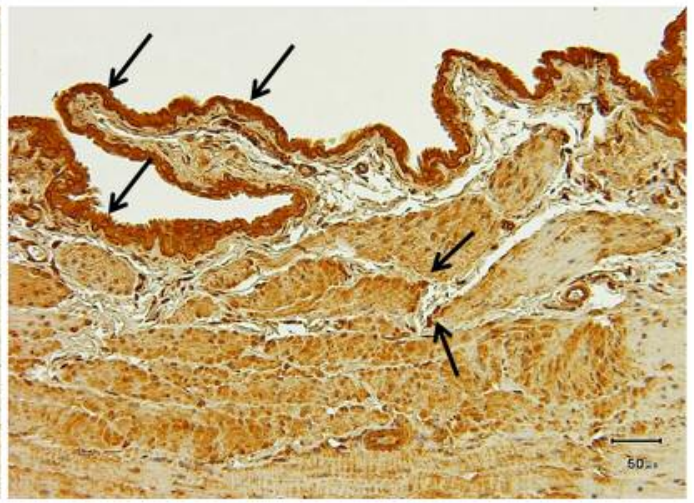

Taurine

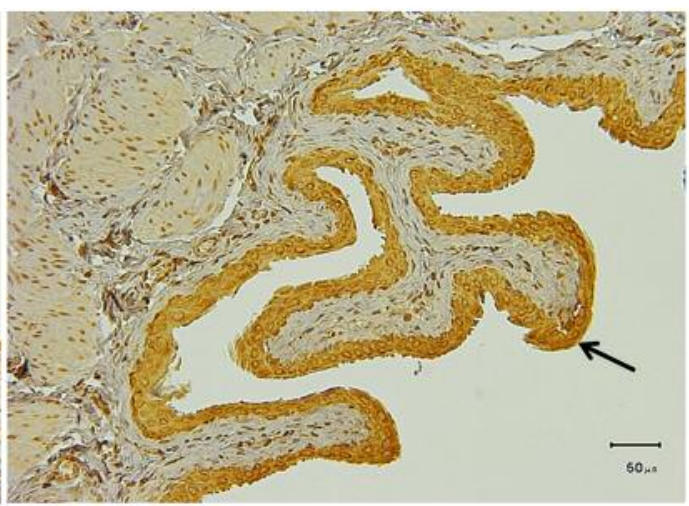

Figure 4. 8-Hydroxy-2-deoxyguanosine, a product of DNA oxidation, is strongly expressed in the urothelium and the muscularis in the bladder of diabetic rats. Arrows indicate 8-hydroxy-2-deoxyguanosine-positive cells stained with brown color. Control group: Fed normal chow and treated with saline daily for 14 days. DM group: Diabetic rats fed with high-fat diet and treated with saline daily for 14 days. Resveratrol group: Diabetic rats fed with high-fat diet and treated with $10 \mathrm{mg} \mathrm{kg}^{-1}$ resveratrol daily for 14 days. Taurine: Diabetic rats fed with high-fat diet and treated with $1 \mathrm{~g} \mathrm{~kg}^{-1}$ taurine daily for 14 days. Original magnification: $\times 200$. Scale bar=50 $\mu \mathrm{m}$.

as oxidative damage at the molecular level can be prevented by treatment with resveratrol or taurine. The present findings in combination with those of previous studies, show that antioxidants may have a beneficial effect on diabetic status and further research is necessary to determine their role as adjunct therapy.

\section{Conflicts of Interest}

The Authors declare that there are no conflicts of interest in regard to this study.

\section{Authors' Contributions}

P.T. Designed the study, performed experiments, analyzed data and wrote the article. M.H. designed the study and critically reviewed the article. K.H. reviewed the experimental protocol. N.S. critically reviewed the article. A.T. critically reviewed the article.

\section{Acknowledgements}

This study was supported by a Grant-In-Aid (KAKENHI, Wakate B) from the Japan Society for the Promotion of Science (17K16793).

\section{References}

1 International Diabetes Federation Guideline Development Group: Guideline for management of postmeal glucose in diabetes. Diabetes Res Clin Pract 103: 256-268, 2014. PMID: 23481145. DOI: $10.1016 /$ j.diabres.2012.08.002

2 WHO: Global Report on Diabetes, 2016. https://apps.who.int/ iris/bitstream/handle/10665/204871/9789241565257_eng.pdf;jse ssionid=289B98D25AAAE2175977686CF30F6044? sequence $=1$

3 Tripathi BK and Srivastava AK: Diabetes mellitus: Complications and therapeutics. Med Sci Monit 12(7): RA130147, 2006. PMID: 16810145 . 
4 Ceriello A and Motz E: Is oxidative stress the pathogenic mechanism underlying insulin resistance, diabetes, and cardiovascular disease? The common soil hypothesis revisited. Arterioscler Thromb Vasc Biol 24: 816-823, 2004. PMID: 14976002. DOI: 10.1161/01.ATV.0000122852.22604.78

5 Brownlee M: Biochemistry and molecular cell biology of diabetic complications. Nature 414: 813-820, 2001. PMID: 11742414. DOI: $10.1038 / 414813 a$

6 Schuller-Levis GB and Park E: Taurine and its chloramine: Modulators of immunity. Neurochem Res 29: 117-126, 2004. PMID: 14992270. DOI: 10.1023/b:nere.0000010440.37629.17

7 Baur JA: Resveratrol, sirtuins, and the promise of a DR mimetic. Mech Ageing Dev 131: 261-269, 2010. PMID: 20219519. DOI: 10.1016/j.mad.2010.02.007

8 Chung JH, Manganiello V and Dyck JR: Resveratrol as a calorie restriction mimetic: Therapeutic implications. Trends Cell Biol 22: 546-554, 2012. PMID: 22885100. DOI: 10.1016/j.tcb. 2012.07.004

9 Olas B, Wachowicz B, Saluk-Juszczak J, Zieliński T, Kaca W and Buczyński A: Antioxidant activity of resveratrol in endotoxin-stimulated blood platelets. Cell Biol Toxicol 17(2): 117-125, 2001. PMID: 11499695.

10 Fang JG, Lu M, Chen ZH, Zhu HH, Li Y, Yang L, Wu LM and Liu ZL: Antioxidant effects of resveratrol and its analogues against the free-radical-induced peroxidation of linoleic acid in micelles. Chemistry 8(18): 4191-4198, 2002. PMID: 12298009. DOI: 10.1002/1521-3765(20020916)8:18<4191::AID-CHEM41 91>3.0.CO;2-S

11 Khalid S, Afzal N, Khan JA, Hussain Z, Qureshi AS, Anwar H and Jamil Y: Antioxidant resveratrol protects against copper oxide nanoparticle toxicity in vivo. Naunyn Schmiedebergs Arch Pharmacol 391(10): 1053-1062, 2018. PMID: 29936585. DOI: $10.1007 / \mathrm{s} 00210-018-1526-0$

12 Dong K, Ni H, Wu M, Tang Z, Halim M and Shi D: ROSmediated glucose metabolic reprogram induces insulin resistance in type 2 diabetes. Biochem Biophys Res Commun 476: 204 211, 2016. PMID: 27207834. DOI: 10.1016/j.bbrc.2016.05.087

13 Palmieri B and Sblendorio V: Oxidative stress tests: Overview on reliability and use. Part II. Eur Rev Med Pharmacol Sci 11(6): 383-399, 2007. PMID: 18306907.

14 Tsounapi P, Honda M, Dimitriadis F, Shimizu S, Hikita K, Muraoka K, Sejima T, Saito M, Tomita S, Sofikitis N and Takenaka A: Post-fertilisation effect of bilateral primary testicular damage induced by unilateral cryptorchidism in the rat model. Andrology 4(2): 297-305, 2016. PMID: 26757429. DOI: 10.1111/andr.12154

15 Ito T, Schaffer SW and Azuma J: The potential usefulness of taurine on diabetes mellitus and its complications. Amino Acids 42: 1529-1539, 2012. PMID: 21437784. DOI: 10.1007/s00726011-0883-5
16 Ikubo N, Saito M, Tsounapi P, Dimitriadis F, Ohmasa F, Inoue S, Shimizu S, Kinoshita Y and Satoh K: Protective effect of taurine on diabetic rat endothelial dysfunction. Biomed Res 32: 187-193, 2011. PMID: 21673448.

17 Tsounapi P, Saito M, Dimitriadis F, Koukos S, Shimizu S, Satoh $\mathrm{K}$, Takenaka A and Sofikitis N: Antioxidant treatment with edaravone or taurine ameliorates diabetes-induced testicular dysfunction in the rat. Mol Cell Biochem 369: 195-204, 2012. PMID: 22763673. DOI: 10.1007/s11010-012-1382-Z

18 Tsounapi P, Honda M, Dimitriadis F, Kawamoto B, Hikita K, Muraoka K, Saito M, Sofikitis N and Takenaka A: Impact of antioxidants on seminal vesicle function and fertilizing potential in diabetic rats. Asian J Androl 19: 639-646, 2017. PMID: 27748317. DOI: $10.4103 / 1008-682 X .186871$

19 Tsounapi P, Honda M, Dimitriadis F, Shimizu S, Shiomi T, Hikita K, Saito M, Tomita S, Sofikitis N and Takenaka A: Antioxidant treatment ameliorates diabetes-induced dysfunction of the vas deferens in a rat model. Andrologia 50(1), 2018. PMID: 28224697. DOI: 10.1111/and.12795.

20 Szkudelski T and Szkudelska K: Resveratrol and diabetes: From animal to human studies. Biochim Biophys Acta 1852: 11451154, 2015. PMID: 25445538. DOI: 10.1016/j.bbadis. 2014.10.013

21 Ustuner MC, Kabay S, Ozden H, Guven G, Yucel M, Olgun EG, Ustuner D, Unal $\mathrm{N}$ and Degirmenci I: The protective effects of vitamin $\mathrm{E}$ on urinary bladder apoptosis and oxidative stress in streptozotocin-induced diabetic rats. Urology 75: 902-906, 2010. PMID: 19683803. DOI: 10.1016/j.urology.2009.06.003

22 Aybek H, Aybek Z, Abban G and Rota S: Preventive effects of vitamin $\mathrm{E}$ against oxidative damage in aged diabetic rat bladders. Urology 77: 508.e10-14, 2011. PMID: 21109295. DOI: 10.1016/j.urology.2010.08.015

23 Ha US, Bae WJ, Kim SJ, Yoon BI, Jang H, Hong SH, Lee JY, Hwang SY and Kim SW: Protective effect of cyanidin-3-O- $\beta-\mathrm{D}-$ glucopyranoside fraction from mulberry fruit pigment against oxidative damage in streptozotocin-induced diabetic rat bladder. Neurourol Urodyn 32: 493-499, 2013. PMID: 23129268. DOI: 10.1002/nau.22334

24 Jiang YJ, Gong DX, Liu HB, Yang CM, Sun ZX and Kong CZ: Ability of alpha-lipoic acid to reverse the diabetic cystopathy in a rat model. Acta Pharmacol Sin 29: 713-719, 2008. PMID: 18501118. DOI: $10.1111 / \mathrm{j} .1745-7254.2008 .00790 . x$

Received September 19, 2019

Revised September 22, 2019

Accepted September 25, 2019 